\title{
Momentum distribution of liquid helium
}

\author{
Saverio Moroni ${ }^{(1)}$, Gaetano Senatore ${ }^{(2,3)}$ and Stefano Fantoni ${ }^{(1,4)}$ \\ (1) International Centre for Theoretical Physics, I-34014, Trieste, Italy \\ (2) Istituto Nazionale di Fisica della Materia \\ (3) Dipartimento di Fisica Teorica, Università di Trieste, Strada Costiera 11, I-34014 Trieste, Italy \\ ${ }^{(4)}$ Interdisciplinary Laboratory, SISSA, Via Beirut 2/4, I-34014, Trieste, Italy
}

(Submitted to Phys. Rev. B, July 18, 1996)

\begin{abstract}
We have obtained the one-body density matrix and the momentum distribution $n(p)$ of liquid ${ }^{4} \mathrm{He}$ at $T=0^{\circ} \mathrm{K}$ from Diffusion Monte Carlo (DMC) simulations, using trial functions optimized via the Euler Monte Carlo (EMC) method. We find a condensate fraction smaller than in previous calculations. Though we do not explicitly include long-range correlations in our calculations, we get a momentum distribution at long wavelength which is compatible with the presence of long-range correlations in the exact wave function. We have also studied ${ }^{3} \mathrm{He}$, using fixed-node DMC, with nodes and trial functions provided by the EMC. In particular, we analyze the momentum distribution $n(p)$ with respect to the discontinuity $Z$ as well as the singular behavior, at the Fermi surface. We also show that an approximate factorization of the one-body density matrix $\rho(r) \simeq \rho_{0}(r) \rho_{B}(r)$ holds, with $\rho_{0}(r)$ and $\rho_{B}(r)$ respectively the density matrix of the ideal Fermi gas and the density matrix of a Bose ${ }^{3} \mathrm{He}$.
\end{abstract}

PACS numbers: 67., 67.40.-w, 67.55.-s

\section{INTRODUCTION}

The momentum distribution $n(p)=<a_{\mathbf{p}}^{\dagger} a_{\mathbf{p}}>$ is a fundamental quantity for the study of both the static and he dynamical properties of quantum liquids, as it gives direct information on the high momentum components of the ground state wave functionl. Experimentally, acCess to $n(p)$ is provided by deep inelastic neutron scattering at large momentum transfer $\hbar Q$. The extraction Of $n(p)$ from the measured scattered intensity, however, - Is'affected by the the limitations imposed by the experimiental resolution and the final-state interactions. Thus, The most accurate information on the momentum distribution of ${ }^{4} \mathrm{He}$ available to date is likely to be the one obJained through accurate, microscopic calculations, such as those presented in this study.

At $T=0^{\circ} \mathrm{K}$ the momentum distribution $n(p)$ of an ideal Bose gas is given by a delta function $\delta(\mathbf{p})$, corresponding to all particle being in the condensate. On the contrary, the condensate fraction of ${ }^{4} \mathrm{He}$ at the equilibrium density is less than $10 \%$, implying that the effects of the strongly repulsive core of the inter-atomic interaction is non perturbative. Similarly for ${ }^{3} \mathrm{He}$ the discontinuity of $n(p)$ at the Fermi momentum $\hbar p_{F}$, which gives the strength of the quasiparticle pole, is $\sim 0.2$ at equilibrium instead of 1, as in the ideal Fermi gas. In fact, it has been always difficult to carry out $a b$ initio calculations of the momentum distribution for these systems, within the field-theoretical approach $\mathrm{B}$.

Modern, realistic, quantitative calculations only started with the development of Variational Monte Carlo (VMC) methods 3 and the Hyper-Netted-Chain (HNC,FHNC) equationst for Jastrow models of both Bose and Fermi liquids. FHNC and HNC equations for the momentum distribution have been numerically solved in Variational calculations of nuclear matterl, liquid ${ }^{4} \mathrm{He}$ 日, and liquid ${ }^{3} \mathrm{He}$, 10, for which triplet and backflow correlations were also taken into account. To improve upon the above variational estimates of $n(p)$, non conventional perturbative techniques, based on correlated basis functions 11 ( $\mathrm{CBF})$, have been deyeloped, and applied to liquid ${ }^{4} \mathrm{He}$ and nuclear matter 6 .

HNC and FHNC theories have the merit to allow for fine details of interparticle correlations, such as longrange behavior, spin dependence, anisotropies in inhomogeneous systems. However, not all cluster diagrams resulting from the theory and involved in the HNC formalism, can be summed in closed form and in FHNC the procedure to estimate the elementary diagrams with exchange bonds is not completely under control. Therefore, approximations like cated summation, 1315 must be invoked, and, in liquid helium calculations, these result in a non negligible loss of acfuracy with respect to a fully variational treatment $16,17$.

The momentum distribution of liquid ${ }^{4} \mathrm{He}$ and ${ }^{3} \mathrm{He}$ has also been calculated, at zero temperature, by ysing the Green Function Monte Carlo (GFMC) method18. finite temperature calculations have been porformed for ${ }^{4} \mathrm{He}$ by Path Integral Monte Carlo (PIMC) 196 and, more recently, by VMC with trial functions of the Shadow type 20 . The available theoretical estimates of the momentum distribution of liquid ${ }^{4} \mathrm{He}$ provided by variational and GFMC methods are in reasonably good agreement among themselves, except for low momenta and for the condensate fraction. For liquid ${ }^{3} \mathrm{He}$ the situation is less satisfactory.

The CFMC and the Diffusion Monte Carlo (DMC) method\$21,22.17, afford to date the most precise tools to perform ground-state calculations for many-particle 
systems. For Bosons they provide estimates of the energy that are virtually exact, within the statistical accuracy. In fact the same is true for the averages of operators that are $(\mathrm{R}-$ ) diagonal (not the case for $n(p)$ !), for which algorithms exist, like the so-called forward walking method23.24, that yield pure estimates. On the other hand, the estimates of observables that do not commute with the Hamiltonian are usually obtained from mixed averages - through an extrapolation procedure whose accuracy depend on the quality of the trial function $\Psi$ used for the importance sampling $\mathrm{B}$. The extrapolation introduces a bias in the estimates which is second order in the difference between $\Psi$ and ground-state wave function $\Phi_{0}$. For Fermions there is an additional source of error related to the existence of the so-called sign problem. To date, to get a numerically stable algorithm it is customary to approximate the unknown nodes of the sought ground state $\Phi_{0}$ with those of the trial function $\Psi$ ( fixed-node approximation 22). This imposes a bias on any average, which for non-diagonal observables cumulates with the one arising from the extrapolation procedure. Therefore, to minimize systematic errors, especially in the evaluation of properties such as the momentum distribution, it is necessary to achieve maximum accuracy in the optimization of the trial function.

Recently, a new optimization procedure based on Monte Carlo calculations and denoted ac Euler Monte Carlo (EMC) method has been proposed25.17. This EMC method has been successfully applied to both liquid ${ }^{4} \mathrm{He}$ and ${ }^{3} \mathrm{He} 6$, 17. The EMC wave functions have pair and triplet correlations fully optimized and provide the lowest available energy upperbounds. Moreover their use in Diffusion Monte Carlo (DMC) calculations21.22] 17 has led to results of unprecedented accuracy for the energy, pair function and static structure function.

In this paper we present results for the one-body density matrix $\rho(r)$ and the momentum distribution $n(p)$ of liquid ${ }^{4} \mathrm{He}$ and ${ }^{3} \mathrm{He}$, at various densities, obtained with DMC calculations based on EMC wave functions. The plan of the paper is as follows. In the next Section we summarize the computational details involved in the calculation of $n(p)$. We then present the results for liquid ${ }^{4} \mathrm{H}$ in Sec. III and those for liquid ${ }^{3} \mathrm{He}$ in Sec. IV. We finally offer a summary and conclusions in Sec. V.

\section{COMPUTATIONAL DETAILS}

In DMC simulations 21.22 the ground state wave functions $\Phi_{0}$ is sampled through a random walk in configuration space, guided by a trial function $\Psi$. In practice one samples the mixed probability $f=\Phi_{0} \Psi$. We chose $\Psi=S F$ for ${ }^{4} \mathrm{He}$ and $\Psi=D_{\uparrow} D_{\downarrow} F$ for ${ }^{3} \mathrm{He}$, with the cor relation part $F$ symmetric in the particle coordinates, $S$ a symmetrized product of one-particle orbitals and $D_{\uparrow}$ and $D_{\downarrow}$ Slater determinants of one-particle orbitals for particles of up and down spin. In the homogeneous liquid $S=$ const and $D_{\sigma}$ is built from plane waves $(\mathrm{PW})$, or from plane waves with short (SBF) or long (LBF) ranged back-flow correctipns. As a full account has already been given elsewhere 19,17 of both the EMC method, which we employ to construct and optimize the trial wave function $\Psi$, and of the use of EMC wave functions in DMC simulations, here we shall restrict to essential details.

All the calculations (variational and diffusion) presented in this paper, unless explicitly noted, have been performed using EMC wave functions with fully optimized pair and triplet pseudopotentials 17 (OJOT)and modeling Helium with the HFDHE2 pair potential of Aziz et al.26. A cubic simulation box and periodic boundary conditions were used. For ${ }^{3} \mathrm{He}$ backflew correlations have been included 17, in the usual way 27 29, by replacing the plane waves $\exp \left(i \mathbf{k}_{i} \cdot \mathbf{r}_{j}\right)$ in the Slater determinants with $\exp \left(i \mathbf{k}_{i} \cdot \mathbf{x}_{j}\right)$, where $\mathbf{x}_{j}=\mathbf{r}_{j}+\sum_{k \neq j} \eta\left(r_{j k}\right)\left(\mathbf{r}_{j}-\mathbf{r}_{k}\right)$. The function $\eta(r)$ is taken either short-ranged 27 (SR)

$$
\eta_{S}(r)=\lambda_{B} \exp \left(-\left(r-r_{B}\right)^{2} / \omega_{B}^{2}\right)((2 r-L) / L)^{3},
$$

or long-ranged 30 (LR)

$$
\eta_{L}(r)=\lambda_{B} \exp \left(-\left(r-r_{B}\right)^{2} / \omega_{B}^{2}\right)+\lambda_{B}^{\prime} / r^{3},
$$

with $\lambda_{B}, r_{B}, \omega_{B}$ and $\lambda_{B}^{\prime}$ variational parameters. The long-ranged backflow function is smoothly cutoff at the the boundary of the simulation box of size $L$ by replacing the expression given in Eq. (2) with $\eta_{L}^{\prime}(r)=\eta_{L}(r)+$ $\eta_{L}(L-r)-2 \eta_{L}(L / 2)$. In practice, we first simultaneously optimize the pair and triplet pseudopotentials with the backflow parameters as specified in Refs. 27 and 30, and then we optimize $\eta(r)$ at fixed pseudopotentials.

The ${ }^{3}$ He results presented below were obtained with short-ranged backflow, unless otherwise specified. Also the DMC simulations were performed within the fixednode approximation, whereby the nodes of the groundstate $\Phi_{0}$ are assumed to coincide with those of the EMC trial function.

In a uniform liquid in a state described by the wave function $\Psi$ the one-body density matrix can be defined as

$$
\begin{aligned}
& \rho(r)= \\
& \frac{V \int d \mathbf{r}_{2} \cdots d \mathbf{r}_{N} \Psi^{\star}\left(\mathbf{r}_{1}, \mathbf{r}_{2}, \cdots, \mathbf{r}_{N}\right) \Psi\left(\mathbf{r}_{1}+\mathbf{r}, \mathbf{r}_{2}, \cdots, \mathbf{r}_{N}\right)}{\int d \mathbf{r}_{1} \cdots d \mathbf{r}_{N}\left|\Psi\left(\mathbf{r}_{1}, \mathbf{r}_{2}, \cdots, \mathbf{r}_{N}\right)\right|^{2}},
\end{aligned}
$$

so that, having imposed periodic boundary conditions to the $N$ particles in the volume $V$,

$$
\rho(0)=1
$$

and the independence on $\mathbf{r}_{1}$ follows from translational invariance. For Fermions, the integration over the variable $\mathbf{r}_{i}$ is understood to imply also the trace over spin projection. If we denote with $R$ and $R^{\prime}$ respectively the configurations $\left(\mathbf{r}_{1}, \mathbf{r}_{2}, \cdots, \mathbf{r}_{N}\right)$ and $\left(\mathbf{r}_{1}+\mathbf{r}, \mathbf{r}_{2}, \cdots, \mathbf{r}_{N}\right)$, and we exploit the translational invariance, we can rewrite Eq. (3) as 


$$
\rho(r)=\frac{\int d R \Psi^{\star}(R) \Psi\left(R^{\prime}\right)}{\int d R|\Psi(R)|^{2}}=\int d R P(R) \frac{\Psi\left(R^{\prime}\right)}{\Psi(R)},
$$

with

$$
P(R)=\frac{|\Psi(R)|^{2}}{\int d R|\Psi(R)|^{2}}
$$

the probability induced by the wave function $\Psi$. Using Eq. (5), the variational density matrix (i.e., the one defined in term of the trial wave function $\Psi$ ) may be conveniently calculated by Monte Carlo as

$$
\rho_{\mathrm{VMC}}(r) \simeq \frac{1}{\mathcal{N}} \sum_{i=1}^{\mathcal{N}} \frac{\Psi\left(R_{i}^{\prime}\right)}{\Psi\left(R_{i}\right)},
$$

with the configurations $R_{i}$ drawn from the probability $P(R)$. The DMC extrapolated estimate $\rho(r)$ is given by

$$
\rho(r)=2 \rho_{\text {mix }}(r)-\rho_{\mathrm{VMC}}(r),
$$

where the mixed estimate $\rho_{\text {mix }}(r)$ is calculated from an expression identical with that of Eq. (7), with the configurations $R_{i}$ drawn however from the mixed probability $P_{\text {mix }}(R)=f(R) / \int d R f(R), f(R)=\Phi_{0}(R) \Psi(R)$. In practice, the auxiliary configurations $R^{\prime}$ appearing in Eq. (7) are generated from a given $R$ by moving a particle either of fixed increments along a random direction (FM) or to points randomly distributed in the simulation box (RM). It turns out that the two methods give more accurate results at small and at large $r$, respectively.

The momentum distribution is defined as $n(p)=$ $\left\langle a_{\mathbf{p}}^{\dagger} a_{\mathbf{p}}\right\rangle$, where an average on spin projections is also implied for unpolarized ${ }^{3} \mathrm{He}$. It is simply related to the one-body density matrix, by a Fourier transform:

$$
\begin{aligned}
n(p) & =\frac{\rho}{\nu} \int d \mathbf{r} e^{i \mathbf{p} \cdot \mathbf{r}} \rho(r) \\
& =\frac{\rho}{\nu}\left(n_{0} \delta(\mathbf{p})+\int d \mathbf{r} e^{i \mathbf{p} \cdot \mathbf{r}}\left(\rho(r)-n_{0}\right)\right),
\end{aligned}
$$

with $\rho=N / V$ the density of the system, $\nu$ the degeneracy factor, which is 1 for ${ }^{4} \mathrm{He}$ and the fully polarized ${ }^{3} \mathrm{He}$, and 2 for normal ${ }^{3} \mathrm{He}$, and $n_{0}$ the large $r$ limit of the density matrix, $n_{0}=\rho(\infty)$. In fact $\rho(r)$ vanishes for large values of $r$ in ${ }^{3} \mathrm{He}$, whereas for ${ }^{4} \mathrm{He}$ it saturates to $n_{0} \neq 0$, due to macroscopic occupation of the state with zero momentum. $n_{0}$ is the condensate fraction, i.e., the fraction of ${ }^{4} \mathrm{He}$ atoms occupying the state with $p=0$.

Evidently, the normalization of the density matrix given in Eq. (4) implies, for the momentum distribution, the normalization sum rule

$$
\frac{\nu}{(2 \pi)^{3} \rho} \int d \mathbf{p} n(p)=\rho(0)=1 .
$$

The momentum distribution has been calculated in two different manners. Having sampled the density matrix as function of $r$ one can just take its Fourier transform according to Eq. (9). Alternatively, restricting to the RM method, one can also directly accumulate

$$
n(\mathbf{p})=\frac{\rho}{\nu} \frac{1}{\mathcal{N}} \sum_{i=1}^{\mathcal{N}} \frac{e^{i \mathbf{p} \cdot \mathbf{r}} \Psi\left(R_{i}^{\prime}\right)}{\Psi\left(R_{i}\right)} .
$$

At a first sight it seems that the $\mathbf{r}$ integration is missing in Eq. (11) above. However, a little reflection shows that accumulating the estimator of Eq. (11) correctly implements the integration (average) over $\mathbf{r}$, which appears in the definition (9) of the momentum distribution, since $\mathbf{r}$ is chosen at random for each configuration $R_{i}$, with a uniform distribution in the simulation box. This second calculation of $n(\mathbf{p})$ is implemented for p's that are reciprocal lattice vectors of the simulation cell. Eq. (11) is employed to accumulate variational and mixed estimators, from which the extrapolated estimator is then obtained, as explained above. From Eqs. (3) it follows that the kinetic energy per particle $T$ can be related to the curvature of the density matrix at the origin, according to

$$
T=-\left[\frac{\hbar^{2}}{2 M} \nabla^{2} \rho(r)\right]_{r=0},
$$

which also implies the kinetic energy sum rule (see Eq. (9))

$$
T=\frac{\hbar^{2}}{2 M} \frac{\nu}{(2 \pi)^{3} \rho} \int d \mathbf{p} p^{2} n(p) .
$$

\section{LIQUID ${ }^{4} \mathrm{HE}$}

We have carried out DMC calculations of the one-body density matrix and of the momentum distribution of ${ }^{4} \mathrm{He}$ at four densities, using EMC trial functions and 64 atoms in the simulation box. Selected runs with up to 232 particles have been performed to check for finite size effects. In Fig. 1 1 we show our results for the one-body density matrix $\rho(r)$ at the equilibrium density. The kinetic energy sum rule (13) is manifestly satisfied and the saturation to a finite $n_{0}$ at large $r$ is evident, in spite of the fact that with 64 particles only distances up to about $7 \AA$ are accessible. We also give in the inset a comparison between estimates obtained with the FM and RM methods. The greater accuracy of the latter method at large distances is apparent. In Fig. 2 we report, also at the equilibrium density, extrapolated estimates of the momentum distribution $n(p)$, obtained using Eq. (11). Due to the finite size of the system only wave vectors larger than $\sim 0.4 \AA^{-1}$ are accessible. We note that a shoulder is discernible in $p n(p)$ at $p \gtrsim 2 \AA$.

In order to extract the condensate fraction $n_{0}$ from our DMC results, as well as to facilitate applications, we have fitted our DMC extrapolated estimates of the density matrix and momentum distribution, obtained with the RM method, to the following analytic formula: 


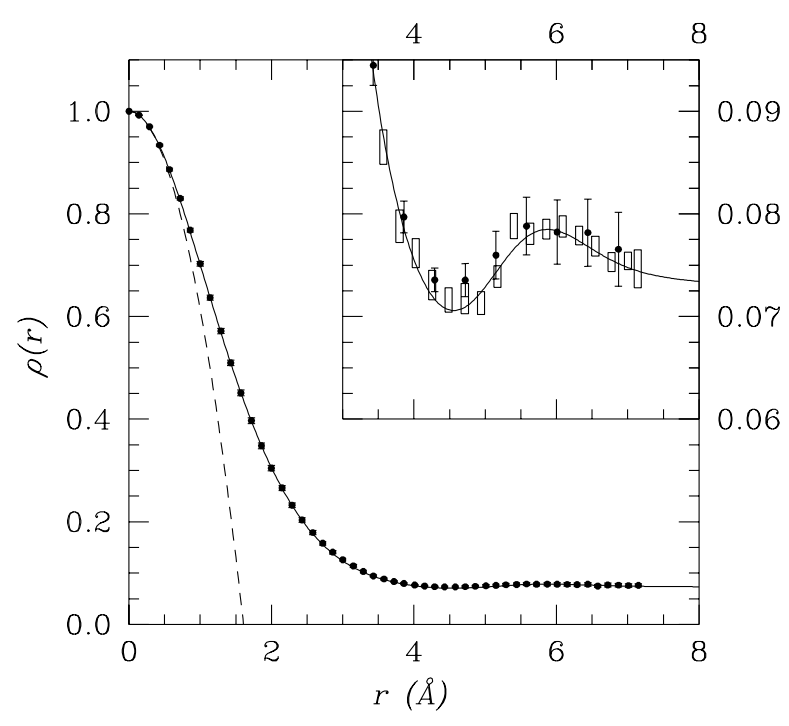

FIG. 1. One body-density matrix of ${ }^{4} \mathrm{He}$ at the equilibrium density $\rho\left(\AA^{-3}\right)=0.02186$. The full curve is the fit of Eq. (14) to our DMC results (extrapolated estimates), with the dashed curve showing the parabola $1-\left(M T / 3 \hbar^{2}\right) r^{2}$ that satisfies the kinetic energy sum rule. The solid circles and rectangles respectively give our DMC results obtained with the FM and RM methods.

$$
\begin{aligned}
n(p)= & (2 \pi)^{3} \rho \delta(\mathbf{p}) n_{0} \\
& +\left(n_{0} \frac{p_{1}}{p}+n_{1} \cos ^{2}\left(\frac{p}{p_{2}}\right)\right) e^{-\left(p / p_{3}\right)^{\alpha}} \\
& +n_{2} e^{-\left(p-p_{4}\right)^{2} / p_{5}^{2}} .
\end{aligned}
$$

The first 2 terms in Eq. (14) account for the existence of the condensate, while the third suitably models the shoulder in $p n(p)$. The fourth and last term accounts for the gross main structure of the momentum distribution.

We have simultaneously fitted $n(p)$ and $\rho(r)$, which must be obtained numerically by Fourier transforming the function of Eq. (14), imposing as well the normalization condition (10) and the kinetic energy sum rule (13). Moreover, we set $p_{1}=M c / 2 \hbar$ to satisfy the long-wavelength behavior

TABLE I. Parameters of the fit (14) to the DMC momentum distribution and density matrix of ${ }^{4} \mathrm{He}$ at $T=0^{\circ} \mathrm{K}$, at various densities. $\rho$ is in $\AA^{-3}$ and $p_{1}-p_{5}$ are in $\AA^{-1}$.

\begin{tabular}{r|llll}
\hline \hline$\rho$ & 0.01964 & 0.02186 & 0.02401 & 0.02622 \\
\hline$n_{0}$ & 0.11163 & 0.071673 & 0.046227 & 0.027079 \\
$\alpha$ & 1.6941 & 1.7634 & 2.2342 & 1.9976 \\
$n_{1}$ & 0.055274 & 0.038282 & 0.019157 & 0.021497 \\
$n_{2}$ & 0.40285 & 0.39893 & 0.36695 & 0.38483 \\
$p_{1}$ & 0.49957 & 0.73394 & 0.95934 & 1.1964 \\
$p_{2}$ & 0.38971 & 0.38525 & 0.40435 & 0.40588 \\
$p_{3}$ & 1.4900 & 1.6744 & 2.2153 & 2.1832 \\
$p_{4}$ & 0.29713 & 0.20538 & 0.28494 & 0.062765 \\
$p_{5}$ & 0.85128 & 1.0016 & 1.0132 & 1.2549 \\
\hline \hline
\end{tabular}

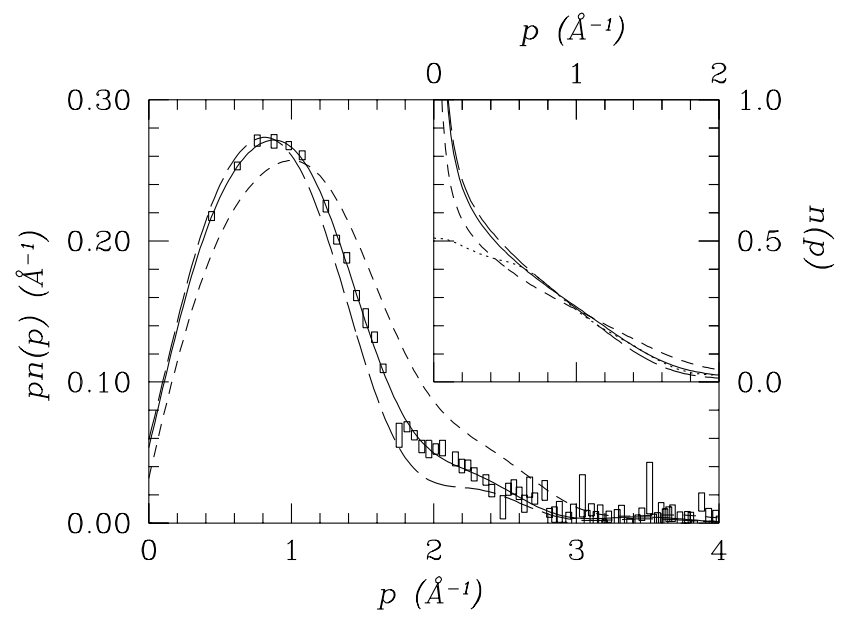

FIG. 2. Momentum distribution of ${ }^{4} \mathrm{He}$ at $T=0^{\circ} \mathrm{K}$. Long dashes, full curve and short dashes are fits to the DMC results at $\rho\left(\AA^{-3}\right)=0.01964,0.02186,0.02622$, whereas the rectangles give the DMC extrapolated estimates at the equilibrium density $\rho\left(\AA^{-3}\right)=0.02186$. The inset shows the long-wavelength behavior ff $n(p)$, with the dotted curve reporting the results of GFMdis.

$$
\lim _{p \rightarrow 0} p n(p)=\frac{n_{0} M c}{2 \hbar},
$$

induced by long-range correlations, as first discussed by Gavoret and Nozieres3. Above $M$ is the mass of the ${ }^{4} \mathrm{He}$ atom and the sound velocity, which we estimate from the DMC17 equation of state (EOS). Thus we fit at each density 6 independent parameters to more than $100 \mathrm{MC}$ points, obtaining a reduced $\chi^{2}$ between 0.98 and 1.20. The resulting fit parameters are recorded in Table I.

In principle one could take $c$ too as unknown and get an independent estimate of the sound velocity. We have tried this alternative, at the equilibrium density, obtaining an estimate of $c$ that is about $10 \%$ lower than experimental and DMC-EOS estimates, and has however a very large uncertainty ( $\sim 50 \%)$, reflecting the absence of DMC points for $p \lesssim 0.4 \AA^{-1}$ ), where the singular term in $n(p)$ is important. We may conclude that our data are compatible with the presence of singular term in $n(p) 31$, in spite of the lack of long-range terms in the pseudopotentials that we have used17. In Fig. 3 we show the density matrix at the four densities that we have studied, as given by the fit of Eq. (14) with the parameters of Table 1 .

DMC momentum distribution and density matrix are compared with the fit of Eq. (14) in Figs. 1, 2, and 4, at the equilibrium density. The fit appears to be very good. A similar conclusion holds at the other densities that we have studied.

The condensate fraction $n_{0}$ is mostly constrained by the large $r$ behavior of $\rho(r)$, which results into the term proportional to $\delta(\mathbf{p})$ in the momentum distribution. As we have already mentioned, the singular behavior of $n(p)$ at small $p$, implied by Eq. (15), is much less effective in 


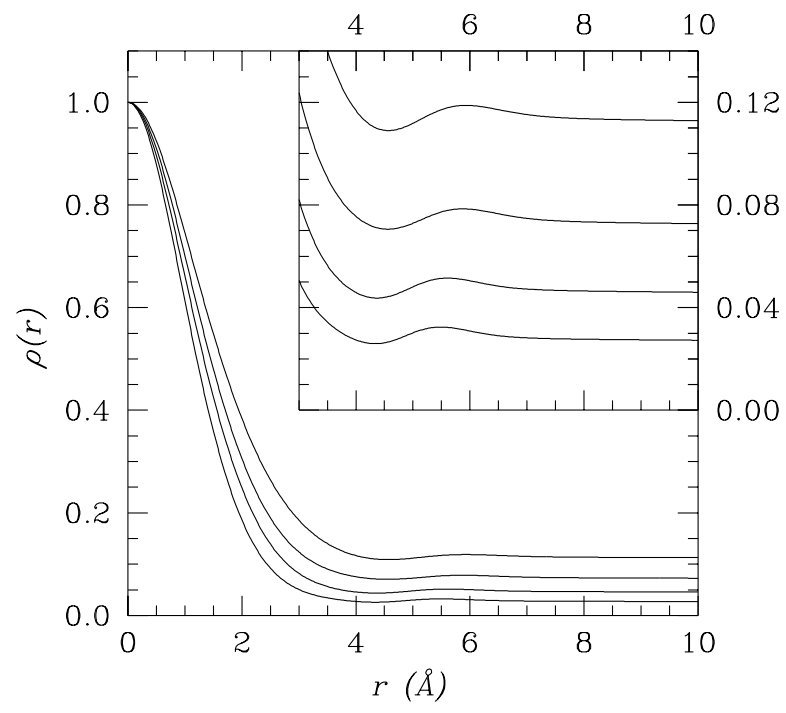

FIG. 3. One-body density matrix $\rho(r)$ of ${ }^{4} \mathrm{He}$. The curves from the topmost to the lowest, give the analytical fit of Eq. (14) to our DMC results (extrapolated estimates), respectively at $\rho\left(\AA^{-3}\right)=0.01964,0.02186,0.02401$ and 0.02622 . The inset shows $\rho(r)$ in the tail region with an enlarged scale.

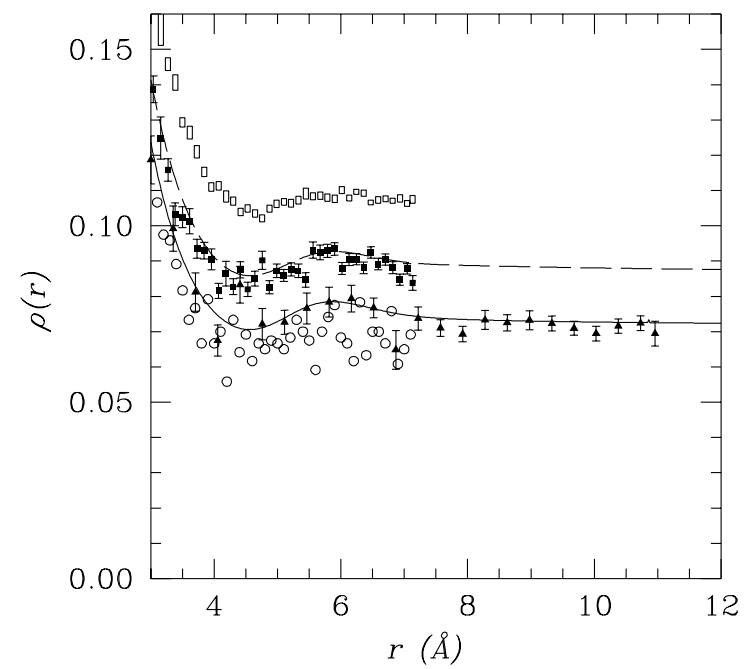

FIG. 4. Dependence on the quality of the wave function and on the method (VMC versus DMC) of the one body-density matrix of ${ }^{4} \mathrm{He}$, at the equilibrium density $\rho\left(\AA^{-3}\right)=0.02186$. The full curve is the fit to our DMC results (extrapolated estimates) for 64 particles, using our best trial function (OJOT), while the dashed curve gives the fit to the VMC results obtained with this trial function. Empty rectangles and solid squares give respectively $\mathrm{VMC}$ and $\mathrm{DMC}$ results obtained from a simple OJ trial function, which embodies only pair pseudopotentials. Finally, the triangles are the DMC results obtained using the OJOT trial function and 232 particles and the circles report the finding of PIMC at $T=1.18^{\circ} \mathrm{K} 1 \mathrm{~g}$.

determining $n_{0}$, because of the absence of DMC estimates for $p \lesssim 0.4 \AA^{-1}$. From the inset of Fig (1) one might conclude that the range $r \lesssim 7 \AA$ accessible with 64 atoms is not big enough too unambiguously assess the value of $n_{0}$. However, simulations with 232 atoms yield, in the extra range $7 \stackrel{⿱}{A} \lesssim r \lesssim 11$, DMC estimates that are in perfect agreement with the fit to the 64 particles results, as it is clear from Fig. A. We also illustrate in this figure the dependence of the large $r$ limit of density matrix, i.e., $n_{0}$, on the quality of the wave function. In particular it is apparent than improving the $\mathrm{MC}$ description either changing from VMC to DMC, for given trial function, or changing to a better trial function in DMC, results into a decrease of $n_{0}$, in the case considered.

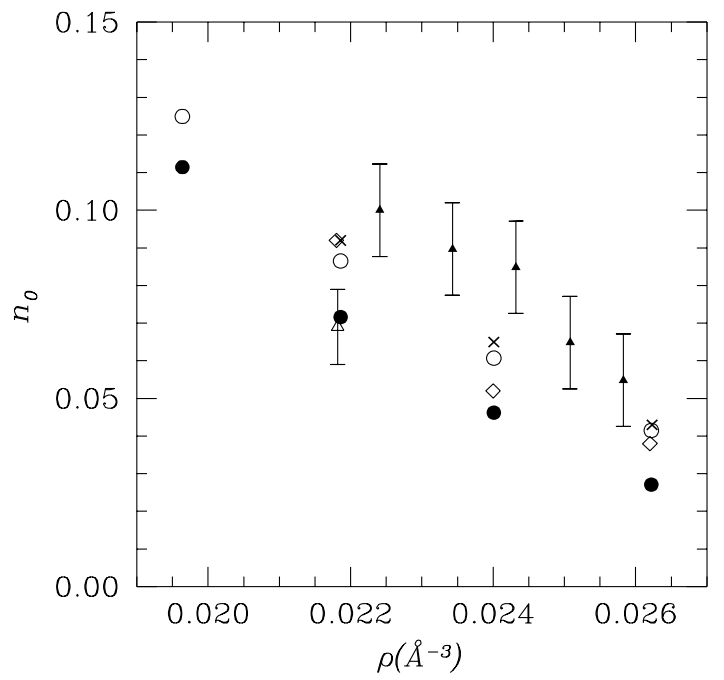

FIG. 5. Condensate fraction of liquid $4 \mathrm{He}$, as a function of the demsity. DMC (solid circles); EMC (open circles); GFMC18 (diamonds); HNCl (crosses); experimental estimates at $T=0.75^{\circ} \mathrm{K} 32$ (solid triangles); PIMC at $T=1.18^{\circ} \mathrm{K} 19$ (empty triangle).

In Fig. 5 and Table [I] we compare our predictions for $n_{0}$ with those from other theoretical treatments as well as with experimental results at low temperature. Consistently with the observation made above, our use of very accurate trial functions 17 yields DMC predictions for the condensate fraction which are-lower than previously obtained by GFMCE and HNCH. On the theoretical side the only prediction that agrees with our own, though it has a much larger statistical error, is the PIMC one at $T=1.18^{\circ} \mathrm{K} 19$. We should remind the reader that in fact PIMC has no trial function bias. We find instead a sizeable discrepancy with the experimental estimates of Snow et al 32 , who determine $n_{0}$ by fitting a model $n(p)$ to the measured Compton profile $J(Y)$ - a procedure however which appears to be model dependent. Different choices for $n(p)$ produce equivalently good fits of $J(Y) 33$, though embodying very different condensate fractions, all the way from $n_{0}=0$ to $n_{0}=10 \%$. In fact, our DMC momentum distribution yields prediction for the Compton profile which agrees well with the experiments, as we show below. 


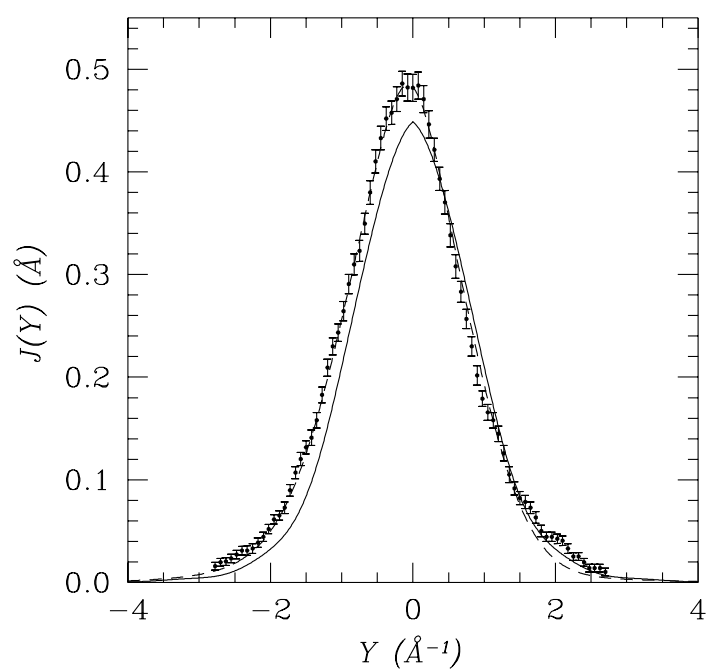

FIG. 6. Compton profile $J(Y)$ of ${ }^{4} \mathrm{He}$ at the equilibrium density $\rho\left(\AA^{-3}\right)=0.02186$ compared with experimental (solid circles) data at $Q=23 \AA^{-1}$ and $T=0.35^{\circ} K{ }^{34}$. The dashed (solid) curve is obtained from our fit to the $\mathrm{DMC} n(p)$ allowing (not allowing) for both the experimental broadening and the final state interactions of Ref. 34. In calculating the dashed curve a shift of $-0.1 \AA$ in $Y$ has been also used as in 34 .

The inelastic neutron scattering cross-section at high momentum transfer $\hbar Q$ can be approximated by its Impulse Approximation (IA) expression, which is proportional to the Compton profile 33

$$
J(Y)=\frac{1}{4 \pi^{2} \rho} \int_{|Y|}^{\infty} d p p n(p) .
$$

The scattering, in IA, does not depend on the energy $\omega$ and the momentum transfer $\hbar Q$ separately, but only through the scaling variable $Y$, given by

$$
Y=(M / \hbar Q)\left(\omega-\omega_{r}\right),
$$

where $\omega_{r}=\hbar^{2} Q^{2} / 2 M$ is the recoil energy of the scattering atom. The dynamical response function $S(Q, \omega)$, in IA, is given by $J(Y)$ times the factor $M /(\hbar Q)$. Final state effects (FSE) of the medium on the scattered atom as well

TABEE II. Confensate fraction $n_{0}$ in ${ }^{4} \mathrm{He}$. DMF GFMC18, and HNd predictions are at $T=0$. The PIMC19 result is at $T=1.18^{\circ} \mathrm{K}$ and the density is in $\AA^{-3}$. The figure in parenthesis is the uncertainty on the last figure, whenever available.

\begin{tabular}{c|llll}
\hline \hline$\rho$ & 0.01964 & 0.02186 & 0.02401 & 0.02622 \\
\hline DMC & $0.112(1)$ & $0.0717(5)$ & $0.0462(6)$ & $0.02 .71(6)$ \\
PIMC & & $0.069(10)$ & & \\
GFMC & & $0.092(1)$ & $0.052(1)$ & $0.037(2)$ \\
HNC & & 0.092 & 0.065 & 0.043 \\
\hline \hline
\end{tabular}

as experimental resolution (ER) broaden up the Compton profile, particularly its delta peak at $p=0$, which is due to the Bose condensation. In Fig. 8 Compton profiles, calculated with our DMC momentum distributions are compared with observed scattering data 34 at $T=0.35^{\circ} \mathrm{K}$, converted to $J(Y)$. Once ER and FSE are taken into account 34 , good agreement with the experiment is obtained.

\section{LIQUID ${ }^{3} \mathrm{HE}$}

For normal ${ }^{3} \mathrm{He}$, DMC simulations using EMC trial functions with backflow and the fixed-node approximation have been performed at five densities, with 54 atoms in the simulation box. We have investigated the dependence of the momentum distribution on the size of the system and on the range of the backflow, respectively with runs for 114 atoms and with runs using trial functions embodying long-range backflow.

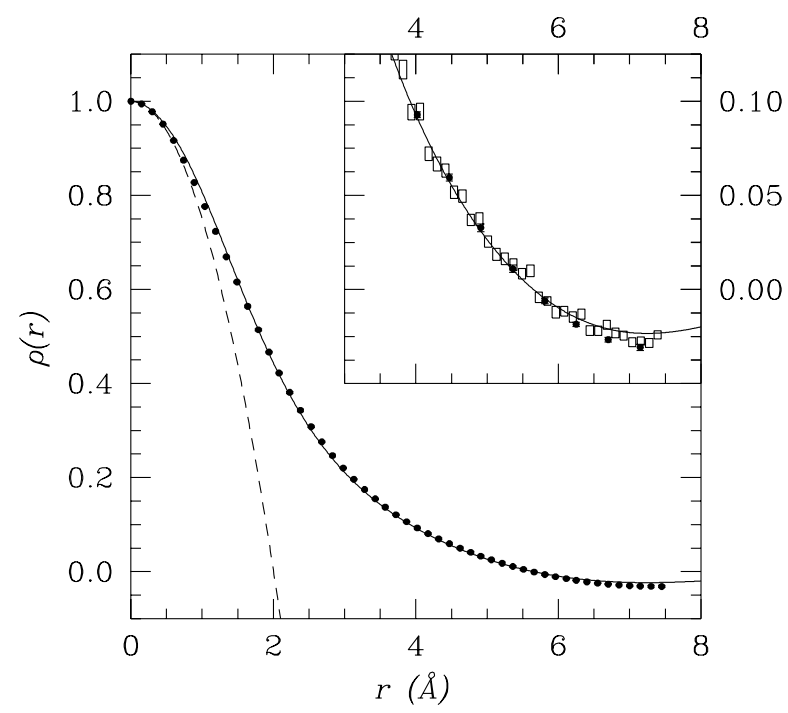

FIG. 7. One body-density matrix of ${ }^{3} \mathrm{He}$ at the equilibrium density $\rho\left(\AA^{-3}\right)=0.01635$. The full curve is the fit of Eq. (19) to our DMC results (extrapolated estimates), with the dashed curve showing the parabola $1-\left(M T / 3 \hbar^{2}\right) r^{2}$ that satisfies the kinetic energy sum rule. The solid circles and rectangles respectively give our DMC results obtained with the FM and RM methods.

In Fig. 7 we give our DMC estimates for the density matrix at the equilibrium density. It is clear that the kinetic energy sum rule (13) is satisfied. The size of the system allows for the determination of $\rho(r)$ through its first zero and up to the first minimum. A comparison between results obtained with FM and RM methods is also given, in the inset. The smaller error on the FM results, compared with that on the RM estimates, is due to the much longer runs used to accumulate the FM $\rho(r)$ in this case. 


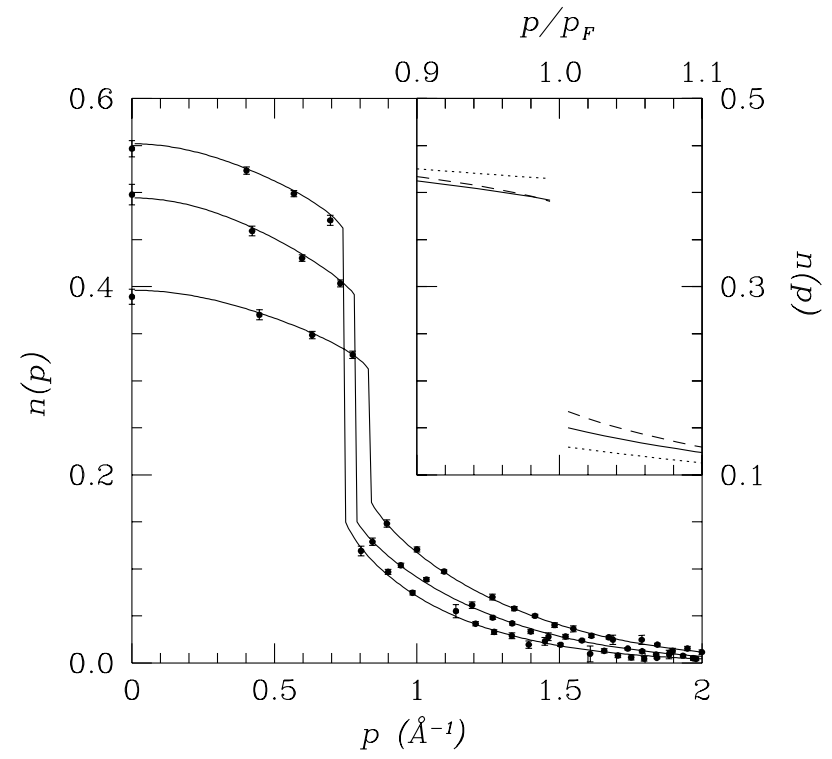

FIG. 8. Momentum distribution of ${ }^{3} \mathrm{He}$ at $T=0^{\circ} \mathrm{K}$ and $\rho\left(\AA^{-3}\right)=0.01413,0.01635$ and 0.01946: dots are DMC (fixed-node) extrapolated estimates; full curves are fits to the DMC results. Larger densities correspond to lower curves at $p=0$. The inset shows the behavior around the Fermi momentum at the equilibrium density $\rho\left(\AA^{-3}\right)=0.01635 \AA^{3}$, using $\mathrm{PW}(\cdots), \operatorname{SBF}(-)$, and $\operatorname{LBF}(--)$ trial functions.

In Fig. 8 we report, at three different densities, the momentum distribution $n(p)$ obtained using Eq. (11). Clearly, the discontinuity $Z$ at the Fermi wave vector $p_{F}$ is substantially reduced, with respect to its value $Z=1$ in the noninteracting system, and moreover it systematically shrinks, as the system gets denser and the effects of the interaction become more important. $\mathrm{Z}$ is also slightly reduced

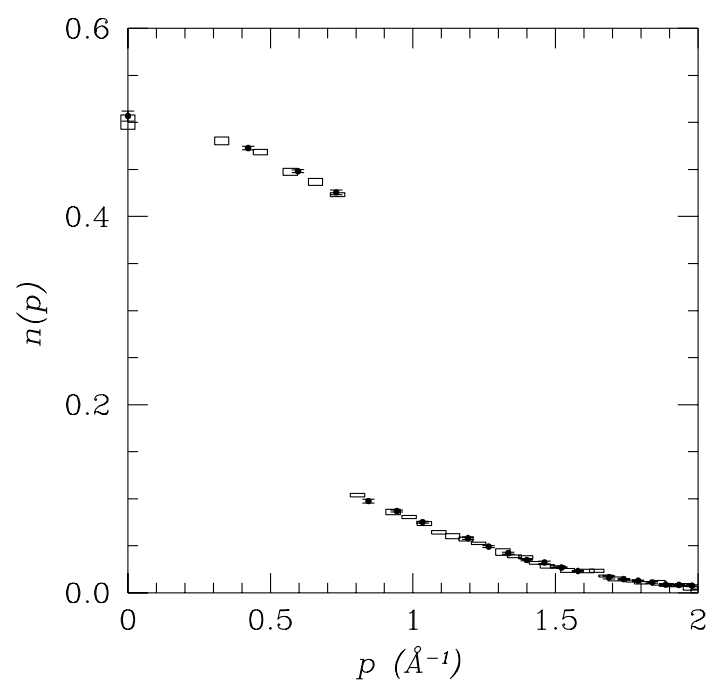

FIG. 9. Momentum distribution of ${ }^{3} \mathrm{He}$ at equilibrium density $\rho\left(\AA^{-3}\right)=0.01635$ with the VMC method, using OJ trial functions, with 54 (solid circles) and 114 (rectangles) particles. when the nodes of the trial function are improved from PW to SRB and then to LBF. Size effects on the momentum distribution appear to be negligible, as it is clear from the comparison between variational results for 54 and 114 particles given in Fig. 9, at the equilibrium density.

In a normal Fermi liquid such as ${ }^{3} \mathrm{He}$ the momentum distributinn, in addition to the discontinuity, has infinite slopes 35,36 at $p_{F}$. To leading order in $p-p_{F}$

$$
n\left(p \rightarrow p_{F}^{ \pm}\right) \simeq n\left(p_{F}^{ \pm}\right)+A \frac{p-p_{F}}{p_{F}} \ln \left|\frac{p-p_{F}}{p_{F}}\right|,
$$

with the coefficient $A$ related to imaginary part of the self-energy $\Sigma(\mathbf{p}, E)$.

To extract $Z$ from the calculated momentum distribution and to check that our results are consistent with the presence of the singular term of Eq. (18) we have fitted our data for $\rho(r)$ and $n(p)$ to the real space form

$$
\begin{aligned}
\rho(r)= & Z \rho_{0}(x)+a_{1} \frac{\rho_{0}(x)}{x}+a_{2} \frac{x \rho_{0}(x)-\sin (x)}{x^{3}} \\
& -a_{1} \frac{e^{-b_{1} x}}{x}+\left(a_{3}+a_{4} x+a_{5} x^{2}+a_{6} x^{3}\right) e^{-b_{2} x}
\end{aligned}
$$

with $x=p_{F} r$, and

$$
\rho_{0}(r)=\frac{3}{x^{3}}(\sin x-x \cos x),
$$

the density matrix of the ideal Fermi Gas. The first two terms in Eq. (19) account respectively for the discontinuity and the infinite slope of $n(p)$ at $p_{F}$. The third term allows for a finite discontinuities in the first and second derivatives of $n(p)$ at $p_{F}$. Finally, the fourth term is needed to eliminate the divergence that the term $\rho_{0}(x) / x$ produces at the origin. We impose the normalization condition (10) and the kinetic energy sum rule (13), as well as the vanishing of the first and third derivative of $\rho(r)$ at $r=0$. Thus we fit at each density 5 independent parameters to more than $100 \mathrm{MC}$ points, with a reduced $\chi^{2}$ between 0.87 and 1.15 . The resulting fit parameters are recorded in Table III.

We compare fit and DMC estimates for $\rho(r)$ and for $n(p)$, respectively in Fig. 7 and Fig. 8. Evidently, Eq. (19) is fully consistent with our DMC results. In Fig. 10 we show the one-body density matrix at three of the densities that we have studied, as given by the fit of Eq. (19). As for the non-interacting case, $\rho(r)$ becomes steeper with increasing the density and its first zero moves toward the origin.

A comparison of our results for the momentum distribution of ${ }^{3} \mathrm{He}$ with those from some other calculations is given in Fig. 11 and in Table [V. HNC results 10 are in close agreement with our variational $n(p)$, for $p \geq p_{F}$ as well as with the estimate of $Z$, while small differences are present at small momenta, which are however of little relevance in the density of states $\propto n(p) p^{2}$. The evident discrepancies between our results and those of GFMC 37 


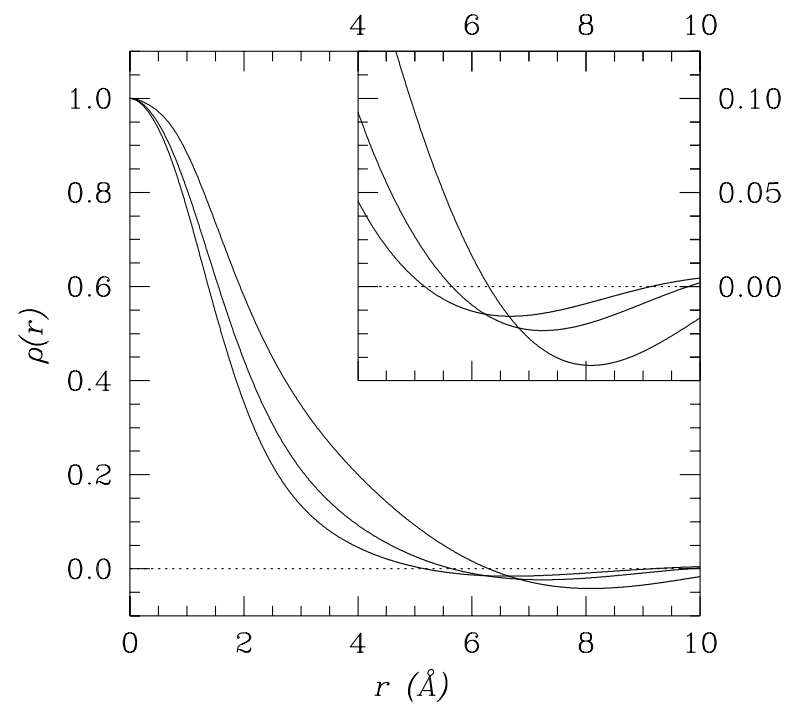

FIG. 10. One-body density matrices $\rho$ of ${ }^{3} \mathrm{He}$. The curves from the less to the most steep, give the analytical fit of Eq. (19) to our fixed-node DMC results (extrapolated estimates), respectively at $\rho\left(\AA^{-3}\right)=0.01198,0.01635$ and 0.01946 . The inset shows $\rho(r)$ in the tail region with an enlarged scale.

around the Fermi surface are probably due to the poorer trial function used in GFMCB7, as well as to statistical errors.

As we have already mentioned, the functional form of Eq. (19) implies for $n(p)$ a singular term at $p_{F}$ of the form (18), with $A=a_{1} / \pi$. For instance, at the equilibrium density $\rho\left(\AA^{-3}\right)=0.01635$ we find $A=0.06(2)$, being however unable at present to assess the size dependence of such estimate. An independent estimate of $A$ is given by a perturbation calculation 36 for a dilute, hard-sphere, Fermi gas. To order $\left(p_{F} R\right)^{2}$,

$$
A_{0}=2 \frac{\nu-1}{\pi^{2}}\left(p_{F} R\right)^{2},
$$

with $R$ the radius of the Fermi particle. Evidently ${ }^{3} \mathrm{He}$ at equilibrium is no at all dilute. Nevertheless, taking $R=\sigma / 2 \simeq 1.3 \AA$, one gets $A_{0} \simeq 0.20$, which is of the same order of magnitude as our DMC estimate. In CBF

TABLE III. Parameters of the fit (19) to the fixed-node DMC momentum distribution and density matrix of ${ }^{3} \mathrm{He}$ at $T=0^{\circ} \mathrm{K}$, at various densities. $\rho$ is in $\AA^{-3}$.

\begin{tabular}{c|ccccc}
\hline \hline$\rho$ & 0.01198 & 0.01413 & 0.01635 & 0.01797 & 0.01946 \\
\hline$Z$ & 0.45977 & 0.30598 & 0.23616 & 0.14328 & 0.13566 \\
$a_{1}$ & 0.14248 & 0.28612 & 0.19376 & 0.41699 & 0.24613 \\
$a_{2}$ & -0.11973 & -0.21912 & -0.12381 & -0.28125 & -0.23982 \\
$a_{3}$ & -0.73200 & -0.80732 & -0.64305 & -2.1780 & -0.96272 \\
$a_{4}$ & 3.2095 & 1.9033 & 3.2723 & 4.1029 & 3.9981 \\
$a_{5}$ & -1.5905 & 0.64801 & -0.098765 & -2.4600 & -0.64725 \\
$a_{6}$ & 7.6229 & 1.5582 & 4.6639 & 6.8026 & 6.2113 \\
$b_{1}$ & 9.0415 & 5.3494 & 7.3460 & 7.3676 & 7.5530 \\
$b_{2}$ & 3.5907 & 2.7487 & 3.0716 & 3.3316 & 3.1652 \\
\hline \hline
\end{tabular}

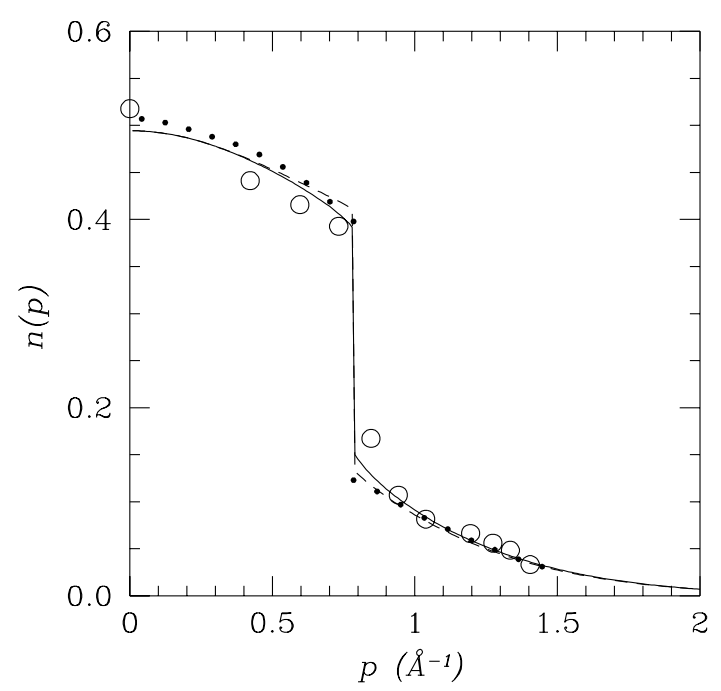

FIG. 11. Momentum distribution of ${ }^{3} \mathrm{He}$ at equilibrium density $\rho\left(\AA^{-3}\right)=0.01635$ : comparison of the present DMC $(-)$ and VMC (- fits with the predictions of GFMCE(circles) and HNCld (solid circles).

theory one obtains 6 , instead,

$$
A_{C B F}=\frac{2 W_{0}}{\pi} p_{F} \frac{d e^{v}\left(p_{F}\right)}{d p},
$$

where $e^{v}(p)$ is the variational energy and $W_{0}$ is an inverse energy parameter characterizing the imaginary part $W(p, E)$ of the self-energy $\Sigma(p, E)$, close to the Fermi energy,

$$
W(p, E) \simeq W_{0}\left(E-e_{F}\right)^{2}, \quad E \rightarrow e_{F} .
$$

If one takes $W_{0} \simeq 2.5^{\circ} K^{-1}$ and $d e^{v}\left(p_{F} / d p=\hbar^{2} p_{F} / M_{v}\right.$, $M_{v} / M=0.76$, from earlier work 38 where a model form of $W(p, E)$ was fitted to the measured specific heat in ${ }^{3} \mathrm{He}$, $A_{C B F} \simeq 21$ is obtained, which is 2 order of magnitude larger than both the DMC and the perturbative estimate.

The effective mass $M^{*}$ is related to the dispersion at $p_{F}$ of the quasi-particle energy

$$
e(p)=\frac{\hbar^{2} p^{2}}{2 M}+\Re \Sigma(p, e(p)),
$$

according to $\hbar^{2} p_{F} / M^{*}=d e\left(p_{F}\right) / d p$. Thus 39.610 $M^{*} / M=M_{E} M_{K}$ with the $\mathrm{K}$-mass

$$
M_{K}^{-1}=1+\left.\frac{M}{\hbar^{2} p_{F}} \frac{\partial}{\partial p} \Re \Sigma(p, E)\right|_{E=e_{F}, p=p_{F}} .
$$

and the $\mathrm{E}$-mass

$$
M_{E}=1-\left.\frac{\partial}{\partial E} \Re \Sigma(\mathbf{p}, E)\right|_{E=e_{F}, p=p_{F}}=Z^{-1} .
$$

Thus, the $\mathrm{E}-$-mass is nothing but $Z^{-1}$, i.e., the strength of the quasi-particle pole at $p_{F}$ en. 41 . We lack, however, 
an estimate of the $\mathrm{K}$-mass, to predict $M^{*}$. Hence, we are planning to perform variational and transient estimate calculations of $M^{*}$, along the lines of an equivalent calculation for the 2-dimensional electron gas 42.43 .

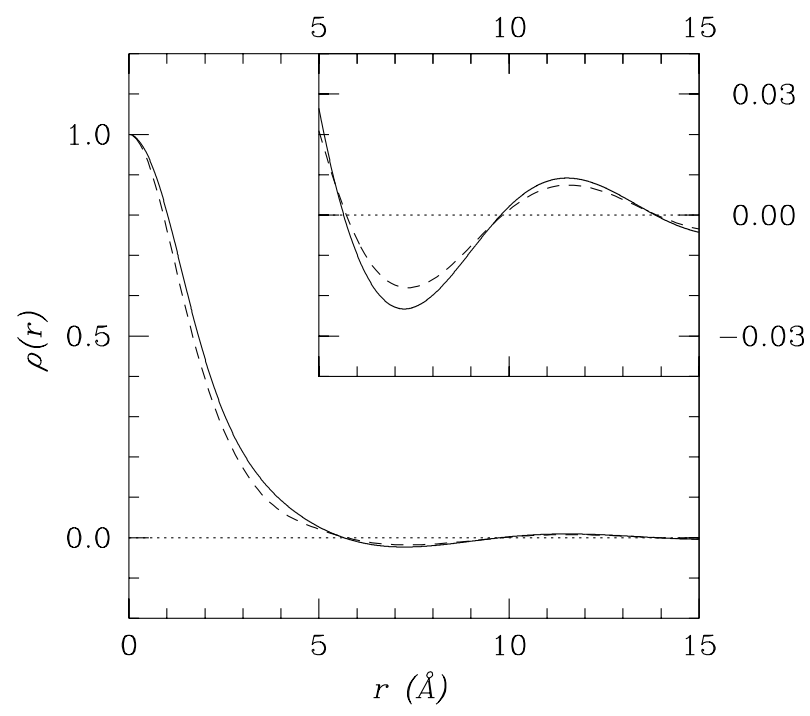

FIG. 12. The one-body density matrix $\rho(r)$ of ${ }^{3} \mathrm{He}$ at $T=0{ }^{\circ} \mathrm{K}$ and $\rho_{e}=0.01635 \AA^{3}$ (full curve). The dashed curve gives the product $\rho_{0}(r) \rho_{B}(r)$, with $\rho_{0}(r)$ and $\rho_{B}(r)$ respectively the density matrix for the ideal (uncorrelated) Fermi gas and the density matrix of a Bose ${ }^{3} \mathrm{He}$.

At the Fermi wave vector $p_{F}$, the momentum distribution $n(p)$ has a discontinuity and, according to perturbation theory and to $\mathrm{CBF}$, at least the additional singular behavior of Eq. (18). It is known 44 that singularities dominate the large distance behavior of the Fourier transform of a generalized function, such as $n(p)$. This imply in particular that as $r \rightarrow \infty \rho(r) \simeq Z \rho_{0}(r)$, to leading order. On the other hand, using the parameters given in Table III one can show that the first zero of the DMC density matrix at the equilibrium density (see, also, Fig. 7) is at $p_{F} r=5.62$, which is not very different from $p_{F} r=5.72$ - the location of the first zero of $\rho_{0}(r)$. We therefore consider the approximate decoupling

$$
\rho(r)=\rho_{0}(r) \rho_{B}(r),
$$

with the function $\rho_{B}(r)$ that must satisfy $\rho_{B}(0)=1$, $\lim _{r \rightarrow \infty} \rho_{B}(r)=Z$, and we further choose it to be everywhere non-negative. It is tempting to take $\rho_{B}(r)$ as the density matrix of a suitable Bose system, with a condensate fraction $Z$.

We have thus simulated a system of ${ }^{3} \mathrm{He}$ with Bose statistics, at the equilibrium density of real ${ }^{3} \mathrm{He}$. We find a condensate fraction of $0.208(5)$, which within error bars agrees with our best estimate of $Z=0.21(2)$. In Fig. 12 we compare our fit to the density matrix of ${ }^{3} \mathrm{He}$ with the prediction of the approximate formula (27), using for $\rho_{B}(r)$ the fit to the simulated density matrix of a Bose ${ }^{3} \mathrm{He}$. It is apparent that the decoupling of Eq. (27) approximately holds. In fact Eq. (27) overestimates the kinetic energy by $14 \%$, while underestimating the envelope of the tail of $\rho(r)$ by about a $20 \%$. Thus statistics and correlations in ${ }^{3} \mathrm{He}$ decouple, within a reasonable accuracy, into those of an ideal (uncorrelated) Fermi gas and a Bose ${ }^{3} \mathrm{He}$, as far as $\rho(r)$ is concerned.

\section{CONCLUSIONS}

We have presented in this paper results of DMC calculations of the one-body density matrix and the momentum distribution of liquid ${ }^{4} \mathrm{He}$ and liquid ${ }^{3} \mathrm{He}$. These DMC simulations are based on accurate trial wave functions with fully optimized pair and triplet pseudopotentials. For ${ }^{3} \mathrm{He}$ the Fermion sign problem has been avoided, by resorting to the fixed-node approximation. In particular, we have used backflow nodes, which are more accurate than the simpler plane-wave nodes. We have recorded our data in a form suitable for future use, in terms of analytical fits.

Our prediction for the condensate fraction of ${ }^{4} \mathrm{He}$ is lower than in previous microscopic calculations and we have argued that these seems to be consistently related to the improved description of ${ }^{4} \mathrm{He}$ afforded by the calculations presented here. We have also found that our results are statistically consistent with the presence of a singular term in $n(p)$ as predicted earlier by Gavoret and Nozieres, in spite of the absence of explicit long-range correlations in our trial functions.

The discontinuity $Z$ of $n(p)$ at $p_{F}$ in ${ }^{3} \mathrm{He}$ is also predicted from our calculations to be sensibly lower than in previous variational calculation and in substantial agreement with GFMC estimates. We have investigate the presence in the $n(p)$ of logarithmic singularities, as predicted by approximate treatments. We find that our results are compatible with the presence of such terms. However the strength of such term is in order of magnitude agreement with perturbation theory, whereas a CBF treatment with empirical parameters for the imaginary part of the optical potential imply a strength which differs from our prediction by 2 orders of magnitude.

We have have also demonstrated that an approximate decoupling $\rho(r) \simeq \rho_{0}(r) \rho_{B}(r)$ holds, with $\rho_{0}(r)$ and

TABLE IV. Discontinuity of the momentum distribution at $p_{F}, Z$, as function of the density, from various calculations. SBF and LBF denote the DMC estimates, obtained using the fit of Eq. (19) and results for EMC wave functions with short and long-range backflow, respectively. VMC gives the variational estimate for the SBF wave function.

\begin{tabular}{c|lllll}
\hline \hline$\rho$ & 0.01198 & 0.01413 & 0.01635 & 0.01797 & 0.01946 \\
\hline HNC $^{\mathrm{a}}$ & & 0.348 & 0.275 & 0.244 & 0.221 \\
VMC & & & $0.272(2)$ & & \\
GFMC $^{\mathrm{b}}$ & & & $\lesssim 0.2$ & & \\
$\mathrm{SBF}$ & $0.46(2)$ & $0.31(2)$ & $0.24(1)$ & $0.14(1)$ & $0.14(1)$ \\
$\mathrm{LBF}$ & & & $0.21(2)$ & & \\
\hline \hline
\end{tabular}

${ }^{\mathrm{a}}$ Ref. 10

${ }^{\mathrm{b}}$ Ref. 18 
$\rho_{B}(r)$ respectively the density matrix for the ideal Fermi gas and the density matrix of a Bose ${ }^{3} \mathrm{He}$. Thus statistics and correlations effects seem to decouple in ${ }^{3} \mathrm{He}$, as far as the density matrix is concerned. The Bose ${ }^{3} \mathrm{He}$ has in fact a condensate fraction which agree within error bars with the discontinuity $Z$ found in the Fermi ${ }^{3} \mathrm{He}$.

We believe that the estimates given in this paper provide the most accurate information of this kind on $\mathrm{He}$ available to date. This is of particular importance for the condensate fraction in ${ }^{4} \mathrm{He}$, as its extraction from deep inelastic neutron scattering seems still not feasible. Using our accurate EMC trial functions we have also studied partially polarized ${ }^{3} \mathrm{He}$. We shall report on this study elsewhere 45 .

\section{ACKNOWLEDGMENTS}

Most of the work presented in this paper was done when SM was enjoying a postdoctoral fellowship at the Laboratorio FORUM of the Istituto Nazionale di Fisica della Materia, Pisa. Access to the computing facilities of CNUCE are also gratefully acknowledged.

${ }^{1}$ Momentum Distributions, R.N.Silver and P.E.Sokol, eds (Plenum, N.Y., 1989).

2 See, e.g., A.L. Fetter and J.D. Walecka, Quantum Theory of Many-Particle Systems (McGraw-Hill, New York, 1971).

${ }^{3}$ D.M.Ceperley and M.H.Kalos, in Monte Carlo Methods in Statistical Physics, K.Binder, ed. (Springer, N.Y., 1979).

${ }^{4}$ S.Fantoni, Nuovo Cimento 44A, 191 (1978).

${ }^{5}$ M.L.Ristig, in From Particle to Nuclei, Course LXXIX Int. School of Phys.Enrico Fermi, A.Molinari ed., (NorthHolland, Amsterdam, 1981)

${ }^{6}$ S.Fantoni and V.R.Pandharipande, Nucl. Phys. A427, 473 (1984).

${ }^{7}$ E.Manousakis, V.R.Pandharipande and Q.N.Usmani, Phys. Rev. B31, 7022 (1985).

${ }^{8}$ A. Fabrocini, S. Rosati, Nuovo Cimento D1, 567, 615 (1982).

9 M.F.Flynn, Phys. Rev. B33, 91 (1986).

${ }^{10}$ A.Fabrocini, V.R.Pandharipande and Q.N.Usmani, Nuovo Cimento 14D, 469 (1992).

${ }^{11}$ E. Feenberg, Theory of Quantum Liquids (Academic, New York, 1969).

12 Q. N. Usmani, S. Fantoni, V. R. Pandharipande, Phys. Rev. B26, 6123 (1982), and references therein.

${ }^{13}$ R. A. Smith, Phys. Lett. 63b, 369 (1976); 85B, 183 (1976); R. A. Smith, A. Kallio, M. Puoskari, P. Toropainen, Nucl. Phys. 328A, 186 (1979).

${ }^{14}$ E. Krotscheck, Phys. Rev. B33, 3158 (1986).

${ }^{15}$ X.Q.G.Wang, S.Fantoni, E.Tosatti and L.Yu, Phys Rev B49, 10027, 1994.

${ }^{16}$ S.Moroni, S.Fantoni, G.Senatore, Europhys. Lett., 30, 93 (1995).
17 S.Moroni, S.Fantoni, G.Senatore, Phys. Rev., B52, 13547 (1995).

18 P.A.Whitlock and R.Panoff, Ca. J. of Phys. 65, 1409 (1987).

19 D.M.Ceperley and E.L.Pollock, Phys. Rev. Lett. 56, 351 (1986); Can J. of Phys. 65, 1416 (1986); E.L.Pollock and D.M.Ceperley, Phys. Rev. B36, 8343 (1986).

${ }^{20}$ G.L.Masserini, L.Reatto, S.A.Vitiello, Phys. Rev. Lett. 69, 2098 (1992).

${ }^{21}$ P.J.Reynolds, D.M.Ceperley, B.J.Alder, W.A.Lester, J. Chem. Phys. 77, 5593 (1982).

${ }^{22}$ D. M. Ceperley, in Recent Progress in Many-Body Theories, ed. J. Zabolitzky (Springer 1981); D. M. Ceperley, J. Stat. Phys. 63, 1237 (1991).

${ }^{23}$ K.S. Liu, M.H. Kalos, and G.V.Chester, Phys. Rev. A10, 303, (1974).

${ }^{24}$ J. Casulleras and J. Boronat, Phys. Rev. B 52, 3654, (1995).

${ }^{25}$ S. A. Vitiello, K. E. Schmidt, Phys. Rev. B46, 5442 (1992).

${ }^{26}$ R. A. Aziz, V. P. S. Nain, J. S. Carley, W. L. Taylor, G. T. Conville, J. Chem. Phys. 70, 4330 (1979).

${ }^{27}$ K. E. Schmidt, M. A. Lee, M. W. Kalos, G. V. Chester, Phys. Rev. Lett. 47, 807 (1981).

${ }^{28}$ E. Manousakis, S. Fantoni, V. R. Pandharipande, Q. N. Usmani, Phys. Rev. B28, 3770 (1983).

${ }^{29}$ Y. Kwon, D. M. Ceperley, R. M. Martin, Phys. Rev. B48, 12037 (1993).

${ }^{30}$ R.M.Panoff, J.Carlson, Phys. Rev. Lett. 62, 1130 (1989).

31 J.Gavoret and P.Noziéres, Ann. Phys. (NY) 28,349 (1964);

${ }^{32}$ W.N.Snow, Y.Wang and P.E.Sokol, Europhys. Lett. 19, 403 (1992)

${ }^{33}$ P.E.Sokol in Bose-Einstein condensation, A.Griffin, D.W.Snoke and S. Stringari, eds. (Cambridge University Press, 1995).

34 T.R.Sosnick, W.M.Snow, P.E.Sokol and R.N.Silver, Europhys. Lett. 9, 707 (1989).

35 S.Fantoni, B.L.Friman and V.R.Pandharipande, Nucl. Phys. A399, 51 (1983).

${ }^{36}$ R.Sartor and C.Mahaux, Phys. Rev. C21, 1546 (1980).

${ }^{37}$ P.E.Sokol, K.Sköld, D.L.Price, R.Kleb, Phys. Rev. Lett. 54, 90 (1985).

38 S.Fantoni, V.R.Pandharipande and K.E.Schmidt, Phys. Rev. Lett. 48, 878 (1982).

39 J.P.Jeukenne, A.Lejeunne and C.Mahaux, Phys.Rep. 25, 83 (1986).

${ }^{40}$ A.B.Migdal, JETP (Sov. Phys) 5, 333 (1957).

${ }^{41}$ J.M.Luttinger, Phys. Rev. 119, 1153 (1960).

${ }^{42}$ Y. Kwon, D.M. Ceperley, and R.M. Martin, Phys. Rev. B50, 1684 (1994).

${ }^{43}$ Y. Kwon, D.M. Ceperley, and R.M. Martin, Phys. Rev. B53 7376 (1996).

${ }^{44}$ M.J. Lighthill, Introduction to Fourier Analysis and Generalized Functions (Cambridge University Press, Cambridge 1959).

${ }^{45}$ S. Moroni, S.Fantoni, G.Senatore, in preparation. 